\title{
Universiteit
}

Leiden

The Netherlands

\section{Dry skin management: practical approach in light of latest research on skin} structure and function

Proksch, E.; Berardesca, E.; Misery, L.; Engblom, J.; Bouwstra, J.A.

\section{Citation}

Proksch, E., Berardesca, E., Misery, L., Engblom, J., \& Bouwstra, J. A. (2019). Dry skin management: practical approach in light of latest research on skin structure and function. Journal Of Dermatological Treatment, 31(7), 716-722. doi:10.1080/09546634.2019.1607024

Version: $\quad$ Publisher's Version

License: $\quad$ Licensed under Article 25fa Copyright Act/Law (Amendment Taverne)

Downloaded from: https://hdl.handle.net/1887/3198869

Note: To cite this publication please use the final published version (if applicable). 


\section{Dry skin management: practical approach in light of latest research on skin structure and function}

Ehrhardt Proksch, Enzo Berardesca, Laurent Misery, Johan Engblom \& Joke Bouwstra

To cite this article: Ehrhardt Proksch, Enzo Berardesca, Laurent Misery, Johan Engblom \& Joke Bouwstra (2020) Dry skin management: practical approach in light of latest research on skin structure and function, Journal of Dermatological Treatment, 31:7, 716-722, DOI: 10.1080/09546634.2019.1607024

To link to this article: https://doi.org/10.1080/09546634.2019.1607024

曲 Published online: 19 Jun 2019.

Submit your article to this journal $\pi$

LII Article views: 835

Q View related articles $\sqsubset$

View Crossmark data ־

4 Citing articles: 9 View citing articles 


\title{
Dry skin management: practical approach in light of latest research on skin structure and function
}

\author{
Ehrhardt Proksch ${ }^{a}$, Enzo Berardesca ${ }^{b}$, Laurent Misery ${ }^{c, d}$, Johan Engblom ${ }^{e}$ and Joke Bouwstra ${ }^{f}$ \\ ${ }^{a}$ Department of Dermatology, University of Kiel, Kiel, Germany; ${ }^{b}$ San Gallicano Dermatological Institute, Rome, Italy; ${ }^{c}$ Department of

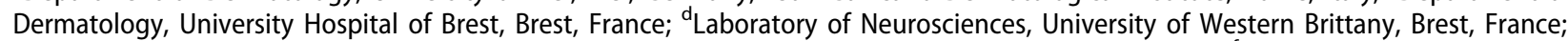 \\ e Department of Biomedical Science, Faculty of Health and Society, Malmö University, Malmö, Sweden; ${ }^{\mathrm{f}}$ Division of Drug Delivery Technology, \\ Leiden Academic Centre for Drug Research, Leiden, The Netherlands
}

\begin{abstract}
Dry skin is a common condition that is attributed to a lack of water in the stratum corneum. With the availability of new technologies, light has been shed on the pathophysiology of dry skin at the molecular level. With the aim to discuss implications of this latest research for the optimal formulation of emollients designed to treat dry skin, five specialists met in November 2017. Research on three topics thereby provided particularly detailed new insights on how to manage dry skin: research on the lipid composition and organization of the stratum corneum, research on natural moisturizing factors, and research on the peripheral nervous system. There was consensus that latest research expands the rationale to include physiological lipids in an emollient used for dry skin, as they were found to be essential for an adequate composition and organization in the stratum corneum but are reduced in dry skin. Latest findings also confirmed the incorporation of carefully selected humectants into a topical emollient for dry skin, given the reduced activity of enzymes involved in the synthesis of moisturizing factors when skin is dry. Overall, the group of specialists concluded that the previous concept of the five components for an ideal emollient for dry skin is well in accordance with latest research.
\end{abstract}

\section{ARTICLE HISTORY}

Received 8 March 2019

Accepted 5 April 2019

\section{KEYWORDS}

Dry skin; skin care; topical; dexpanthenol; position paper

\section{Introduction}

During the past decade, considerable progress has been made in our understanding of the pathophysiology of dry skin conditions, thanks to new technologies that allowed us to evaluate dry skin at the molecular level. Although most research has focused on dry skin related to skin diseases such as atopic dermatitis, findings also led to an improved understanding of the development and composition of dry skin when occurring as a condition itself $(1,2)$. Thus, there exist data that provide a strong rationale for the optimal ingredients needed in a formulation designed to treat dry skin. To date, formulations for the treatment of dry skin often contain ingredients for which rationales are weak and also no clear evidence of benefits exists. It is, therefore, time to link new research on dry skin, molecular research in particular, to the composition of emollient formulas, to optimize treatment of the symptom affecting nearly everybody of us to a more or less extent. Against this background, a group of specialists in the field of dry skin convened in November 2017. Research on three topics thereby provided particularly detailed new insights on how to manage dry skin: research on the lipid structure and organization of the stratum corneum (SC), research on natural moisturizing factors (NMFs), and research on the peripheral nervous system.

\section{What is dry skin?}

Dry skin (xerosis) is characterized by rough, scaly, or flaky skin and is accompanied by loss of skin elasticity and frequently pruritus. It is a common symptom of a number of skin conditions, such as atopic dermatitis, ichthyosis, irritant contact dermatitis, psoriasis or asteatotic eczema (eczema craquelé), but it may also represent a condition itself $(1,3,4)$. The onset of dry skin is thought to be mediated by genetics, environmental factors, ageing and other factors such as ethnicity.

\section{Roles of genetics and environmental factors}

From the genetic perspective, dry skin is likely to be the result of a number of mutations across different pathways. Some of these pathways are related to certain dermatological conditions. For instance, atopic dermatitis and ichthyosis vulgaris are associated with mutations to the gene coding for the structural protein filaggrin. A deficiency in filaggrin, and its degradation products which are part of the NMF, is associated with dry skin $(1,5)$. NMF is a mixture of hygroscopic compounds important for maintaining adequate skin hydration. Other mutations associated with dry skin conditions involve the involucrin gene or certain aquaporin genes $(6,7)$.

Environmental factors, such as frequent washing/showering or exposure to low air humidity, are also triggers for dry skin. As an example, xerosis is more prevalent in the winter season when the air is at low humidity. The latter creates a water gradient between the skin and the external environment; this increases the driving force to draw water out of the skin and the skin becomes drier (8). When coupled with low grade inflammation, dry skin may transition into eczema. 


\section{Role of ageing}

Aging makes skin more susceptible to dryness. There are various potential etiologies of dry skin in older people including reduced epidermal differentiation and lipid synthesis (3). Additionally, the level of filaggrin is lower in aged skin (7). As a result, the skin's water holding capacity decreases significantly.

\section{Role of body location}

Certain body sites show an increased risk of becoming very dry. For example, skin on the fingertips and knuckles are often exposed to high levels of mechanical stress and lack skin flexibility; both of these factors contribute to the higher level of skin dryness often observed at these skin sites. Also, the face and the extremities are prone to dry skin because of a higher curve radius compared to trunk which enhances water evaporation. In addition, the face is usually unprotected by cloth and exposed to the environment.

\section{Role of ethnicity}

Ethnicity primarily refers to the observable differences in the physical appearance of individuals of different racial groups. However, establishing a significant set of differences that distinguishes one racial group from another proves difficult, since genetic variation between individuals of the same racial group can often be greater than the genetic variation observed between individuals of different racial groups. Generally, individuals can be classified into four racial groups: white, black, Hispanic, and Asian.

Although some physiological skin differences exist between ethnic groups, such as number of SC layers, transepidermal water loss (TEWL), or SC $\mathrm{pH}$, the similarities prevail (9-12). The likelihood of developing dry skin in individuals of different racial groups is primarily driven by environmental factors (e.g. climatic conditions) rather than ethnicity.

\section{Healthy skin is an equilibrium}

The SC is key in regulating water flux and retention. There are several processes involved in the formation and effective function of the SC which include NMF formation, lipid processing, keratinocyte differentiation, and desquamation (i.e. loss of corneocytes, shedding occurs within 2 weeks in normal SC) $(3,13)$. An adequate equilibrium is necessary between these four, highly interdependent processes to keep the skin healthy. Figure 1 illustrates a subset of key events affecting skin equilibrium, with the caveat that other crucial points are not shown for reasons of clarity, such as $\mathrm{pH}$, role of stress hormones, or the influence of the gut microbiome on skin homeostasis (14).

Environmental factors are also important in influencing this equilibrium. It is assumed that these environmental triggers act in two steps: first short-term effect, then on a more long-term perspective through the perturbation of normal epidermal differentiation (i.e. the stepwise transformation of keratinocytes into corneocytes). The short-term effect can be illustrated by the consequences when the skin is suddenly exposed to cold, dry air: water is quickly lost from the SC (owing to the altered water gradient). As short-term consequence, there is insufficient water for a proper functioning of enzymes involved in NMF production and epidermal desquamation.

The longer-term effect of exposure to adverse environmental conditions (such as cold air with low humidity) results in major perturbation of normal epidermal differentiation which ultimately ends in a dry skin cycle (Figure 2) as described by Rawlings (15). Within the dry skin cycle, the skin barrier attempts to repair itself

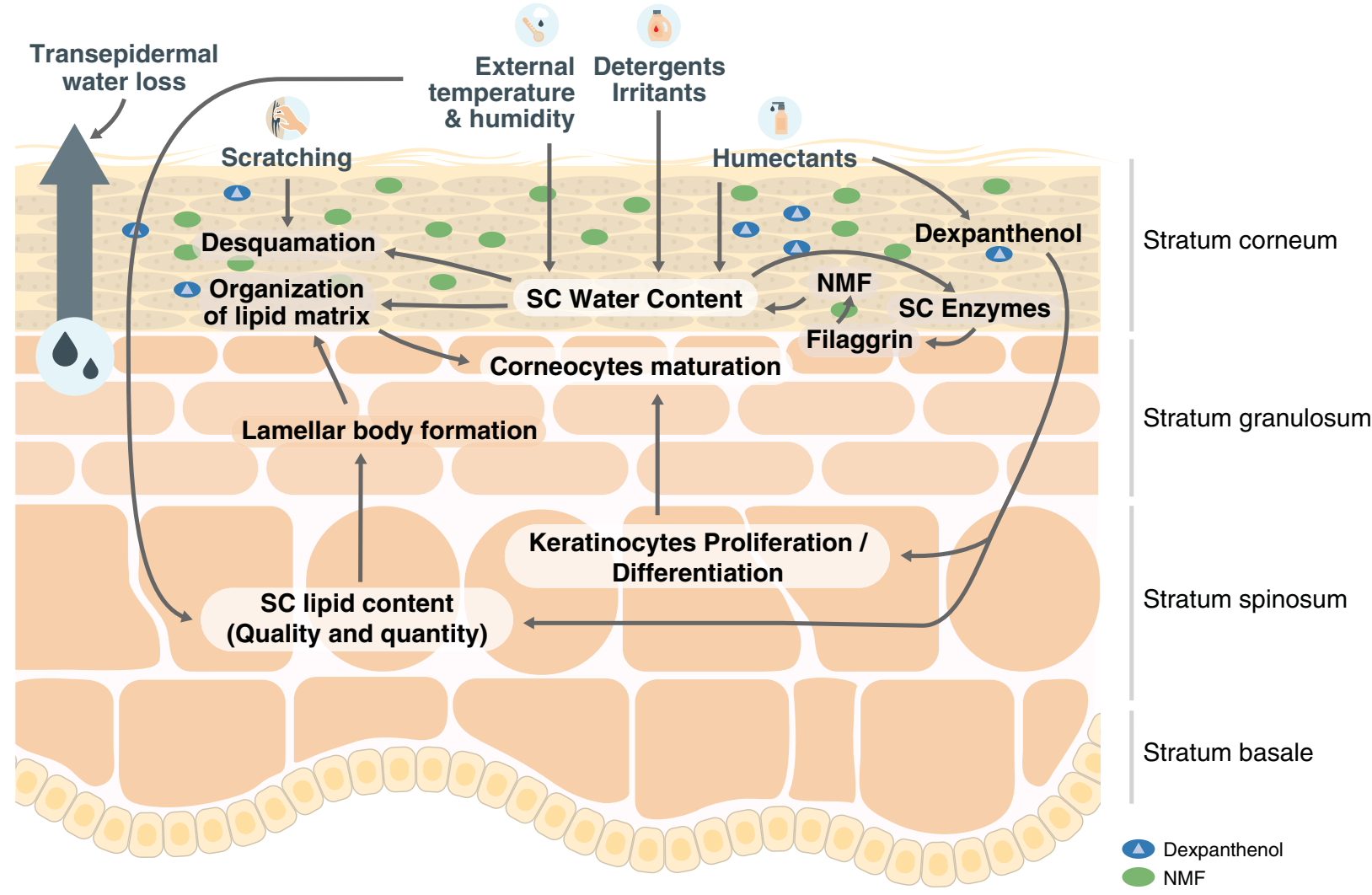

Figure 1. Schematic diagram showing a subset of key events affecting skin equilibrium. 
which induces a hyperproliferative state, characterized by dysfunctional keratinocyte differentiation. In turn, this results in further impairment to maturation and desquamation of the SC, thereby becoming thicker with reduced water-binding properties. The induction of the inflammatory hyperproliferative state is key in the cycle of dry skin as it leads to production of small/immature

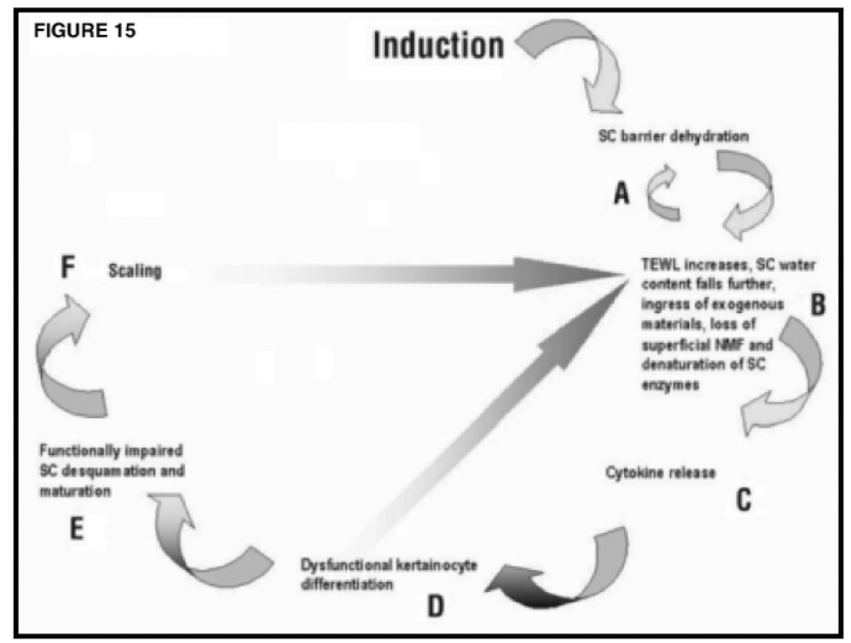

Figure 2. Schematic diagram showing pivotal events within the dry skin cycle. From Ref. (15) with kind permission from Elsevier Inc. NMF: natural moisturizing factor; SC: stratum corneum; TEWL: transepidermal water loss. corneocytes and overproduction of a variety of poor quality cellular materials and structures vital to the proper functioning of the skin barrier (15).

\section{Dry skin at the molecular level}

\section{Research on SC lipids structure and organization}

Research on atopic skin, notably non-lesional skin may be used as a proxy for dry skin in otherwise healthy individuals. A crucial factor for the skin barrier function is the lipid matrix in the SC. The composition of the SC lipid matrix is dominated by three lipid classes: ceramides, cholesterol, and free fatty acids (2). Physiologically, they are present in an approximately equimolar ratio and show a highly ordered, 3-dimensional structure of stacked densely packed lipid layers (lipid lamellae); the lipid composition determines how these lipids are ordered $(2,16)$. The lipids can be assembled in an orthorhombic, hexagonal, or liquid lateral organization. Orthorhombic packing is the most prevalent in healthy human skin and is considered to be important for the barrier function (17-19). When viewed from the perspective of lamellar organization as analyzed by X-ray diffraction, two distinct phases can be identified based on repeat distances of approximately $6 \mathrm{~nm}$ and $13 \mathrm{~nm}$, forming the short and long periodicity phases, respectively (Figure 3 ). The long periodicity phase is thought to play a role for proper barrier function in healthy skin $(2,20)$.

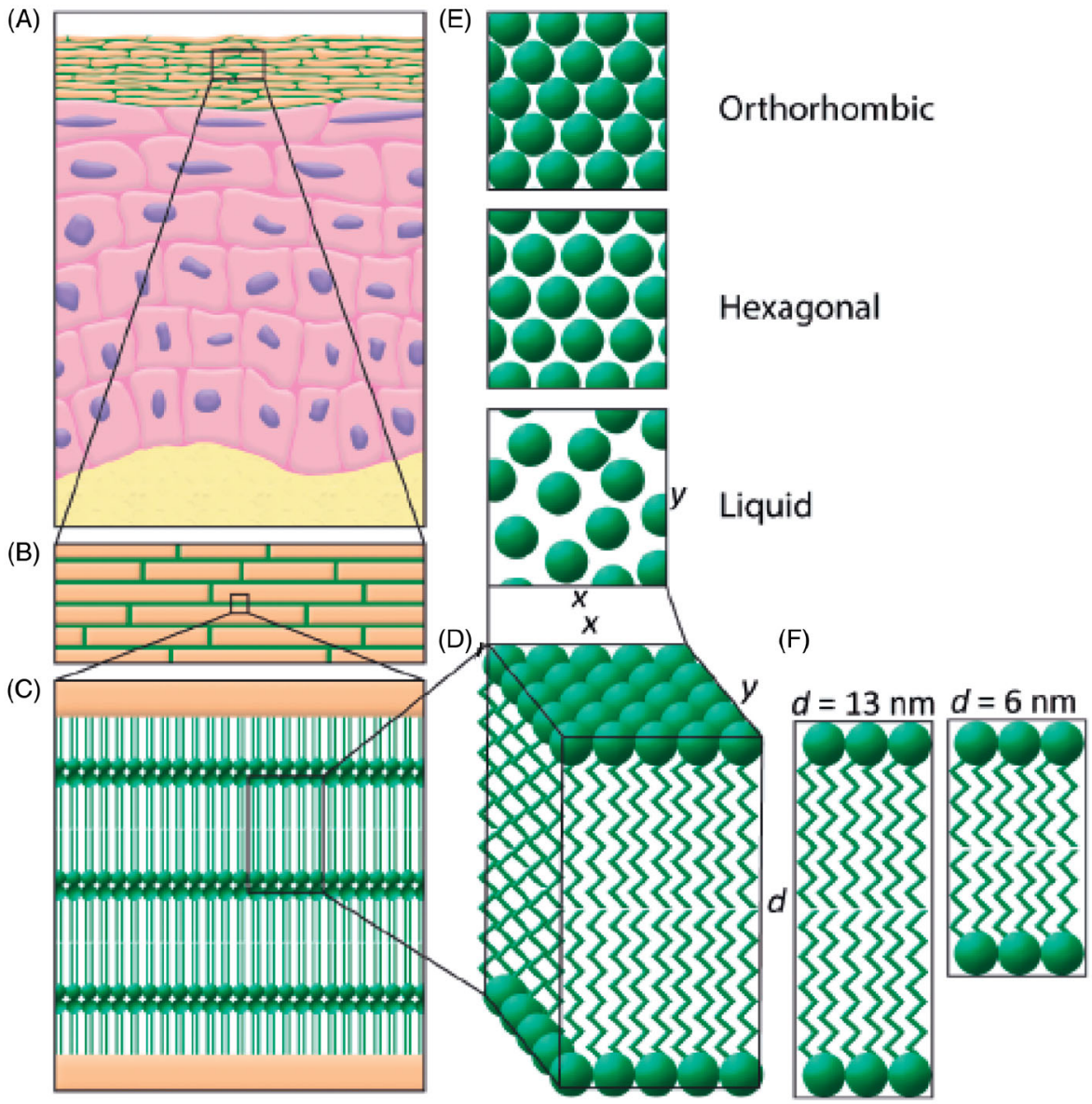

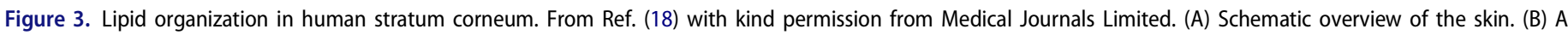

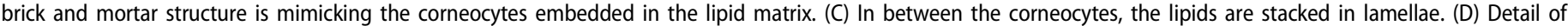

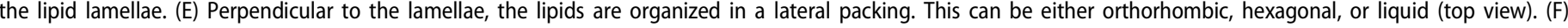
The lipid lamellae are stacked on top of each other with a repeat distance (d) of either $13 \mathrm{~nm}$ (long periodicity phase) or $6 \mathrm{~nm}$ (short periodicity phase). 
Skin barrier at reduced water activity

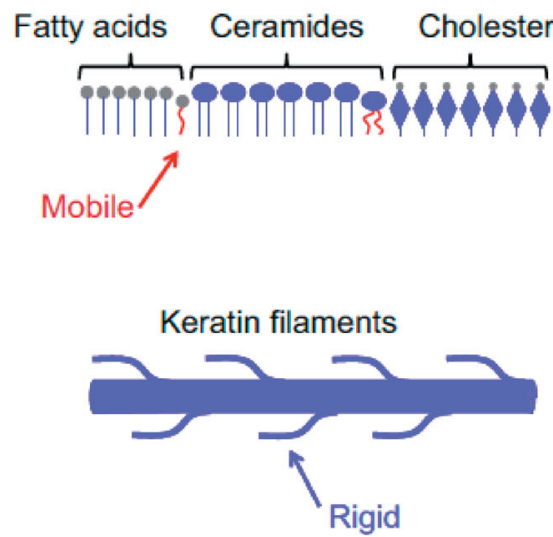

Skin barrier at reduced water activity with transcutol or dexpanthenol

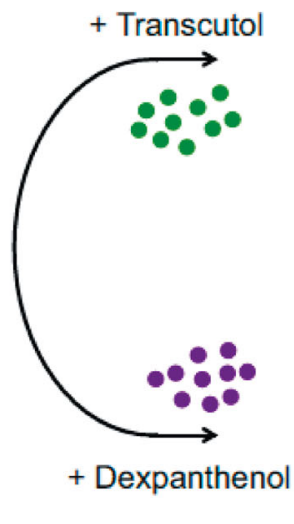

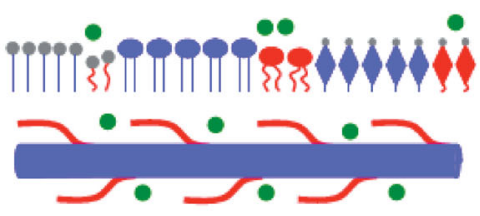

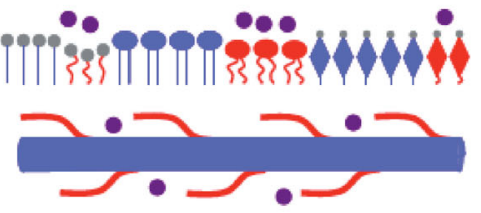

Figure 4. Influence of dexpanthenol and transcutol on membrane fluidity. From Ref. (27) with kind permission from Elsevier Inc.

Using non-lesional atopic dermatitis as a model for dry skin, distinct ceramide, and free fatty acid profiles (characterized by lipid molecules with shorter chain lengths), as well as an abnormal lipid organization (with an increased lipid fraction forming a hexagonal packing and lipids in lamellar phases with a reduced repeat distance), are observed compared with healthy skin $(16,21-23)$. It may, therefore, be inferred that the impaired skin barrier function (monitored by transepidermal water loss) observed in dry skin conditions is associated with two main alterations in SC lipid composition/organization. First, there is an increased level of lipids with shorter chain length in both the ceramide and free fatty acid lipid classes. In fact, lipid chain length reduction correlates with a less dense lipid organization and a decreased skin barrier function (23). The longer chains allow for stronger intermolecular forces between the lipid chains than the intermolecular forces for shorter chains. Second, increased levels of SC lipids adopting a hexagonal packing at the expense of the orthorhombic packing enhance impairment of skin barrier function.

Apart from lipid organization and composition, the total SC lipid content is considered to be another variable for effective skin barrier function. In lesional and non-lesional SC of subjects with atopic dermatitis, a reduced lipid/protein ratio has been observed which correlated strongly with skin barrier function (i.e. increase in TEWL) and disease severity (24).

The changes in SC lipids may contribute to the understanding of risk factors for dry skin. For instance, in healthy subjects it was shown that the SC quantities of ceramides, free fatty acids, and cholesterol are reduced during winter compared with the summer season. In addition, significantly decreased levels of all major lipid classes, in particular ceramides, are observed with increasing age (25).

\section{Research on NMFs and humectants}

Since years, humectants have been used to replace lost NMFs in situation of dry skin. There is scientific evidence that humectants can replicate NMF function and are capable of correcting defects in skin elasticity (7). Hence, a humectant should be part of an emollient formula designed to combat dry skin, even more because it has been shown that humectants are able to attenuate the hyperproliferative response to barrier disruption (26).
Recent research on compounds used traditionally as humectants has revealed that not only they act by binding water into the SC but also influence lamellar lipids organization. Björklund and colleagues showed that hygroscopic dexpanthenol and transcutol generate the properties of a hydrated SC in dehydrated conditions (27). Both substances have similar physicochemical properties as urea and glycerol and it has been suggested that these molecules influence the molecular dynamics of the SC in a similar manner, with the difference that dexpanthenol makes both lipids and keratin filaments more fluid than transcutol (Figure 4) (27). Specifically, it could be shown that dexpanthenol interacts with lipid segments of the extracellular lamellae and protein residues in the corneocytes of SC and thus compensates for reduced hydration by retaining/increasing molecular mobility/fluidity $(27,28)$. By this mechanism, dexpanthenol reduces the increased rigidity of the SC lipid lamellae and proteins (keratin filaments) observed in dry skin.

In conclusion, healthy skin is an equilibrium that can easily be disturbed by intrinsic and external factors. If dry skin has developed, it is critical that the condition is managed effectively and early to avoid short-term and long-term consequences, with the primary focus at restoring the skins equilibrium.

\section{How to restore skin's equilibrium? Practical implication for formulation}

Dry skin management typically involves application of an emollient. It has been previously recommended that an ideal emollient contains five key components: humectants, non-physiological lipids (e.g. mineral, vegetable oil), physiological lipids (e.g. ceramides, free fatty acids), antipruritics/soothing agents, and agents that support epidermal differentiation (3). Here we review the results from recent studies on humectants, skin lipids and peripheral nervous system and their implication for the selection of the ingredients for dry skin management.

\section{Contribution of skin lipids research}

When interpreting new scientific findings, it becomes obvious that there is accumulating evidence that SC lipids play an important role in dry skin conditions. Topical emollients for barrier function recovery should therefore include lipids. From an efficacy point of view, physiological lipids would be the preferred choice. 
For testing formulations designed for skin barrier repair, a novel ex vivo human model has been recently developed in which the SC is removed by a reproducible stripping technique (29). Using this model, 20 strips of SC were taken from healthy individuals following topical ceramide application. After 20 strips, the ceramide that had been applied was still detectable in the SC. However, in recent studies, it could not be determined whether this is present in furrows or penetrated into the SC (30). The value of physiological lipids has also been demonstrated in studies that used topical formulations which were based on vernix caseosa, a white cheesy film found on the surface of an infant's skin during gestational delivery. Vernix caseosa contains the three physiological barrier lipids identified in the human skin. A formulation that mimicked the composition of vernix caseosa was applied to ex vivo skin. The fatty acids present in the formulation were shown to intercalate with the endogenous skin barrier lipids and to enhance the fraction of lipids forming an orthorhombic (very dense) packing in the SC (17). Therefore, physiological lipids can make a valuable contribution to emollient preparations.

\section{Implications for optimal dry skin management}

New scientific findings provide a strong rationale for the incorporation of lipids into topical emollients to be used for dry skin management. For normalization of SC lipid composition and organization (predominantly the reconstitution of the orthorhombic laterally packed phase and the $13 \mathrm{~nm}$ long periodicity lamellar phase), physiological lipids, such as ceramides or long chain free fatty acids, are the preferred choices. However, there might be cost implications upon use of these compounds. Mineral and vegetable oils are affordable alternative options but they work primarily via an occlusive effect and are not expected to exert their action by partitioning into the SC lipid matrix. Nevertheless, by staying on the SC surface, they provide beneficial effects by working like a temporary dressing. In future, ingredients that target enzymes responsible for the synthesis of specific lipids or for elongation of lipids present in the SC may be selected to address alterations in the SC lipid organization/composition.

\section{Contribution of research on humectants}

The selected humectant has ideally a molecular weight of 200-300 Da (in any case $\leq 500 \mathrm{Da}$ ) to ensure that the hygroscopic compound is able to penetrate the skin surface and reach the deeper layers of the SC, where it can exert its required function. Larger molecules, such as hyaluronic acid, are unable to penetrate the skin but can exert their benefits at the skin surface level by retaining moisture within the formulation after application to skin (31). From an efficacy point of view, glycerol and urea (a natural component of NMF but not a degradation product of filaggrin) are still the preferred choices for humectants. Both are small molecules and represent physiological humectants that play a key role in keeping the SC hydrated (7). Urea is more effective than glycerol when the SC water content is low (32) but physical properties (e.g. sensitive to heat, $\mathrm{pH}$-dependent stability, prone to precipitation) may be problematic for emollient manufacturing. In addition, urea has to dissolve to exert its action. Thus, in very dry conditions, urea may not work. Upon inclusion of a humectant as an ingredient in topical emollients, it is critical that the humectant is used at an optimal concentration. For example, if a formula contains too much glycerol, it will dry the skin owing to its hygroscopic nature (33).

Compounds that can work as humectants but reveal additional features, such as dexpanthenol, are also an option to consider for inclusion in topical emollients. Apart from acting as a humectant, dexpanthenol stimulates intracellular protein and lipid synthesis, increases fibroblast proliferation, and enhances epidermal differentiation $(7,28)$.

\section{Implications for optimal dry skin management}

A humectant should be part of an emollient formula designed to manage dry skin conditions. Newer research findings confirm that there is a compelling scientific rationale for the currently used humectants. Physiological humectants, such as glycerol and urea, are the preferred choices when used at the right concentration. However, humectants that incorporate additional functions are also viable options for inclusion in topical emollients.

\section{Contribution from peripheral nervous system research}

Itching is a common symptom of both sensitive skin and dry skin, which may be particularly pronounced when both conditions are present simultaneously. A history of atopic dermatitis is observed in $20-30 \%$ of subjects with sensitive skin (34). Sensitive skin has been defined by the occurrence of unpleasant sensations (stinging, tingling, burning, pain, pruritus) in response to stimuli that normally should not provoke such sensations. These unpleasant sensations cannot be explained by lesions attributable to any skin disease; the skin can appear normal or be accompanied by erythema. Sensitive skin can affect all body locations, especially the face (35). Sensitive skin is not limited to individuals with dry skin but should be considered in that context because dry and sensitive skin is often combined, and sensitive skin has similar trigger factors. For instance, unpleasant sensations in sensitive skin can be caused by physical stimuli, such as cold/hot weather, wind, air conditioning, or UV exposure. There are also chemical (e.g. cosmetic ingredients, soaps, pollution), psychological, and hormonal trigger factors (35).

The pathophysiology of sensitive skin has not been fully elucidated. However, the occurrence of unpleasant sensations implies that the nervous system is involved. Although the density of intraepidermal nerve fibers is reduced in sensitive skin compared with non-sensitive skin, they appear to generate a greater number of sensorineural impulses via the action of transient receptor potential (TRP) channel proteins. TRP proteins are expressed in various tissues including the skin (36) where they are the only proteins known to be activated by physical and chemical factors (37). The increase in sensorineural impulses is reflected by unpleasant sensations. Some TRP channel proteins can be activated by ingredients encountered in cosmetic products leading to skin irritation (38). It is, therefore, important to exclude potential TRP protein activators (e.g. polyunsaturated fatty acids) and to carefully select the ingredients when formulating products for sensitive skin (with or without concomitant dry skin). One of the ingredients should provide relief from itching.

\section{Implications for optimal dry skin management}

Sensitive skin is probably caused by neuronal mechanisms. For defining nerve fiber endings as therapeutic target, the scientific knowledge is currently insufficient, despite the advances made in elucidating the pathomechanisms involved in sensitive skin. To combat itching, which is common in both sensitive and dry skin (and is particularly pronounced if both conditions are combined), an antipruritic agent (e.g. glycine, glycyrrhetinic acid, polidocanol) should be part of an ideal topical emollient. It helps to break the self-perpetuating itching-scratching cycle. 
Table 1. Key ingredients to be included in topical emollients for management of dry skin [modified from (3)].

\begin{tabular}{|c|c|}
\hline Ingredient & Rationale \\
\hline $\begin{array}{l}\text { Humectants (e.g. glycerol, urea, propylene glycol, hya- } \\
\text { luronic acid) }\end{array}$ & $\begin{array}{l}\text { If the humectant is of small size (MW }=200-300 \mathrm{Da}) \text {, such as glycerol or urea, deeper layers of SC are } \\
\text { reached where they restore water content and barrier function, and replicate NMF function. Larger } \\
\text { molecules (e.g. hyaluronic acid) do not penetrate the skin but increase the hydration of the outermost } \\
\text { corneocytes }\end{array}$ \\
\hline Non-physiological lipids (e.g. mineral, vegetable oil) & $\begin{array}{l}\text { Act as a substitute for lost natural skin lipids in the outer layers of the SC. Reduce TEWL by creating a } \\
\text { hydrophobic barrier on the skin surface (occlusive effect) }\end{array}$ \\
\hline Physiological lipids (e.g. ceramides, free fatty acids) & $\begin{array}{l}\text { Penetrate the SC effectively and contribute to normalization of the altered lipid composition and organiza- } \\
\text { tion (e.g. reconstitution of the lipid fraction forming an orthorhombic [very dense] packing). Are impli- } \\
\text { cated in maintaining the structural elements of the SC and may mediate the stimuli that trigger } \\
\text { epidermal differentiation }\end{array}$ \\
\hline Antipruritics (e.g. glycine)/soothing agents & Prevent or relieve itching. Break the self-perpetuating itching-scratching cycle \\
\hline $\begin{array}{l}\text { Multifunctional component including enhancement of } \\
\text { epidermal differentiation (e.g. dexpanthenol) }\end{array}$ & $\begin{array}{l}\text { Apart from acting as a humectant, dexpanthenol stimulates protein/lipid synthesis and supports epidermal } \\
\text { regeneration by enhancing epidermal differentiation. Dexpanthenol compensates for reduced hydration } \\
\text { by retaining/increasing molecular mobility/fluidity of the SC lipid lamellae and proteins }\end{array}$ \\
\hline
\end{tabular}

MW: molecular weight; NMF: natural moisturizing factor; SC: stratum corneum; TEWL: transepidermal water loss.

\section{Conclusions}

The concept of the inclusion of five components in an ideal emollient for dry skin (non-physiological lipids, physiological lipids, humectants, antipruritics/soothing agents, and agents supporting epidermal differentiation) remains valid, but the scientific rationale has been expanded (Table 1). In this paper, we discuss the outcome of studies shedding new light on skin lipids and humectants at the molecular level providing a scientific rationale for the presence of these compounds in a formulation designed to treat dry skin.

Physiological lipids (in particular ceramides and free fatty acids with longer chains) are required for an adequate composition and organization (orthorhombic packing) of the SC lipid matrix but their amount is reduced in dry skin. Physiological lipids as part of an emollient preparation may enhance the fraction of lipids forming an orthorhombic (very dense) packing in the SC, thereby improving skin barrier function in conditions of dry skin. The mineral and vegetable oils (i.e. non-physiological lipids) that provide an occlusive effect on the skin's surface represent another important component of topical emollients for dry skin. Given that enzymes involved in the synthesis of NMF and epidermal desquamation do no properly work when skin is dry, humectants are also relevant in a topical emollient used to treat dry skin. Although nerve fiber endings have been identified as the site where pruritus is initiated, regardless of skin hydration status, targeting involved proteins is not possible today. Thus, topical emollients for dry skin should contain an antipruritic/soothing agent.

The specialist panel also confirmed the important role of dexpanthenol in topical emollients for dry skin management. Among its multiple functions, dexpanthenol compensates for reduced hydration by increasing water content and by favorably influencing the molecular mobility/fluidity of the SC lipid lamellae and proteins. Another key feature of dexpanthenol is its beneficial effect on epidermal regeneration due to an enhancement of epidermal differentiation. By supporting the epidermal regeneration process, dexpanthenol contributes to barrier function recovery in dry skin conditions and helps to reestablish normal epidermal differentiation. The latter feature is important to address the longterm issues associated with dry skin and to interrupt the dry skin cycle.

To combat dry skin even more efficiently in future, research on substances that target enzymes responsible for the synthesis of lipids deficient in dry skin (particularly ceramides and free fatty acids) or for elongation of lipids still present in the SC should be intensified.
Table 2. Galenical formulation to be selected for dry skin management by season and body site affected [modified from (3)].

\begin{tabular}{ll}
\hline Season/body site & \multicolumn{1}{c}{ Formulation } \\
\hline Winter & Ointment rich in lipids \\
Summer & Cream rich in water \\
Extremities & Ointment rich in lipids \\
Face & Cream rich in water \\
\hline
\end{tabular}

The environment plays a greater role in dry skin onset than genetic factors or ethnicity. Moreover, depended on the body site, the skin lipid content varies; the face and abdominal areas show larger amounts of SC lipids than skin on leg and palmoplantar areas (3). Therefore, the emollients necessary to manage dry skin should be available in different formulations that can be selected according to an individual's environmental condition (season, climate) and skin site affected (Table 2). This approach can also be applied to strategies to manage dry skin in young versus aged skin (with creams preferred for the former, and ointments for the latter) (39).

Considering the latest findings on dry skin and their implications for designing an optimal topical emollient for the treatment of dry skin, it will now be time to put the gained insights into practice by adapting or creating new formulations for the combat against a condition which affects most of us at some time.

\section{Disclosure statement}

Ehrhardt Proksch and Laurent Misery have previously participated in specialist panels organized by Bayer.

\section{Funding}

Bayer Consumer Care AG organized and funded the specialist panel meeting.

\section{References}

1. Danby SG. Biological variation in skin barrier function: from A (atopic dermatitis) to $X$ (xerosis). Curr Probl Dermatol. 2016;49:47-60.

2. van Smeden J, Bouwstra JA. Stratum corneum lipids: their role for the skin barrier function in healthy subjects and atopic dermatitis patients. Curr Probl Dermatol. 2016;49: 8-26. 
3. Proksch E, Lachapelle JM. The management of dry skin with topical emollients - recent perspectives. J Deut Dermatol Gesell. 2005;3:768-774.

4. Moncrieff G, Cork M, Lawton S, et al. Use of emollients in dry-skin conditions: consensus statement. Clin Exp Dermatol. 2013;38:231-238.

5. Elias PM, Hatano Y, Williams ML. Basis for the barrier abnormality in atopic dermatitis: outside-inside-outside pathogenic mechanisms. J Allergy Clin Immunol. 2008;121: 1337-1343.

6. Sevilla LM, Nachat R, Groot KR, et al. Mice deficient in involucrin, envoplakin, and periplakin have a defective epidermal barrier. J Cell Biol. 2007;179:1599-1612.

7. Giménez-Arnau A. Standards for the protection of skin barrier function. Curr Probl Dermatol. 2016;49:123-134.

8. Stick C, Proksch E. The influence of climate on the treatment of dry skin with moisturizer. In: Lodén $M$, Maibach $H$, editors. Treatment of dry skin syndrome. Berlin, Heidelberg: Springer; 2012. p. 503-511.

9. Kompaore $\mathrm{F}$, Tsuruta $\mathrm{H}$. In vivo differences between Asian, black and white in the stratum corneum barrier function. Int Arch Occup Environ Heath. 1993;65:S223-S225.

10. Berardesca $E$, Pirot $F$, Singh $M$, et al. Differences in stratum corneum $\mathrm{pH}$ gradient when comparing white Caucasian and black African-American skin. $\mathrm{Br} J$ Dermatol. 1998;139: 855-857.

11. Voegeli R, Rawlings AV, Seroul $P$, et al. A novel continuous colour mapping approach for visualization of facial skin hydration and transepidermal water loss for four ethnic groups. Int J Cosmet Sci. 2015;37:595-605.

12. Wiznia LE, Elbuluk N. Differences in skin structure and function in ethnic populations. In: Vashi NA, Maibach HI, editors. Differences in skin structure and function in ethnic populations. New York: Springer International Publishing AG; 2017. p. $35-48$.

13. Kraft JN, Lynde CW. Moisturizers: what they are and a practical approach to product selection. Skin Ther Lett. 2005;10: $1-8$.

14. Salem I, Ramser A, Isham N, et al. The gut microbiome as a major regulator of the gut-skin axis. Front Microbiol. 2018;9: 1459.

15. Rawlings AV, Matts PJ. Stratum corneum moisturization at the molecular level: an update in relation to the dry skin cycle. J Invest Dermatol. 2005;124:1099-1110.

16. Moore DJ, Rawlings AV. The chemistry, function and (patho)physiology of stratum corneum barrier ceramides. Int J Cosmet Sci. 2017;39:366-372.

17. Berkers T, van Dijk L, Absalah S, et al. Topically applied fatty acids are elongated before incorporation in the stratum corneum lipid matrix in compromised skin. Exp Dermatol. 2017;26:36-43.

18. Berkers T, Visscher D, Gooris GS, et al. Degree of skin barrier disruption affects lipid organization in regenerated stratum corneum. Acta Derm Venereol. 2018;98:421-427.

19. van Smeden J, Janssens M, Gooris GS, et al. The important role of stratum corneum lipids for the cutaneous barrier function. Biochim Biophys Acta. 2014;1841:295-313.

20. de Jager M, Groenink W, Bielsa i Guivernau R, et al. A novel in vitro percutaneous penetration model: evaluation of barrier properties with p-aminobenzoic acid and two of its derivatives. Pharm Res. 2006;23:951-960.
21. van Smeden J, Hoppel L, van der Heijden R, et al. LC/MS analysis of stratum corneum lipids: ceramide profiling and discovery. J Lipid Res. 2011;52:1211-1221.

22. Janssens $M$, van Smeden J, Gooris $G S$, et al. Increase in short-chain ceramides correlates with an altered lipid organization and decreased barrier function in atopic eczema patients. J Lipid Res. 2012;53:2755-2766.

23. van Smeden J, Janssens $M$, Kaye EC, et al. The importance of free fatty acid chain length for the skin barrier function in atopic eczema patients. Exp Dermatol. 2014;23:45-52.

24. Janssens $M$, van Smeden J, Puppels GJ, et al. Lipid to protein ratio plays an important role in the skin barrier function in patients with atopic eczema. Br J Dermatol. 2014;170: 1248-1255.

25. Rogers J, Harding C, Mayo A, et al. Stratum corneum lipids: the effect of ageing and the seasons. Arch Dermatol Res. 1996;288:765-770.

26. Denda $M$, Sato J, Tsuchiya $T$, et al. Low humidity stimulates epidermal DNA synthesis and amplifies the hyperproliferative response to barrier disruption: implication for seasonal exacerbations of inflammatory dermatoses. J Invest Dermatol. 1998;111:873-878.

27. Björklund S, Pham QD, Jensen LB, et al. The effects of polar excipients transcutol and dexpanthenol on molecular mobility, permeability, and electrical impedance of the skin barrier. J Colloid Interface Sci. 2016;479:207-220.

28. Proksch E, de Bony $\mathrm{R}$, Trapp $\mathrm{S}$, et al. Topical use of dexpanthenol: a 70th anniversary article. J Dermatolog Treat. 2017; 28:766-773.

29. Danso MO, Berkers T, Mieremet $A$, et al. An ex vivo human skin model for studying skin barrier repair. Exp Dermatol. 2015;24:48-54.

30. Boiten WA, Berkers T, Absalah S, et al. Applying a vernix caseosa based formulation accelerates skin barrier repair by modulating lipid biosynthesis. J Lipid Res. 2018;59:250-260.

31. Albèr $C$, Brandner BD, Björklund $S$, et al. Effects of water gradients and use of urea on skin ultrastructure evaluated by confocal Raman microspectroscopy. Biochim Biophys Acta. 2013;1828:2470-2478.

32. Albèr C, Buraczewska-Norin I, Kocherbitov V, et al. Effects of water activity and low molecular weight humectants on skin permeability and hydration dynamics - a double-blind, randomized and controlled study. Int J Cosmet Sci. 2014;36: 412-418.

33. Caussin J, Groenink HW, de Graaff AM, et al. Lipophilic and hydrophilic moisturizers show different actions on human skin as revealed by cryo scanning electron microscopy. Exp Dermatol. 2007;16:891-898.

34. Richters R, Falcone D, Uzunbajakava N, et al. What is sensitive skin? A systematic literature review of objective measurements. Skin Pharmacol Physiol. 2015;28:75-83.

35. Misery L, Ständer S, Szepietowski JC, et al. Definition of sensitive skin: an expert position paper from the special interest group on sensitive skin of the international forum for the study of itch. Acta Derm Venerol. 2017;97:4-6.

36. Duarte I, Silveira J, Hafner MFS, et al. Sensitive skin: review of an ascending concept. An Bras Dermatol. 2017;92:521-525.

37. Tóth BI, Oláh A, Szöllősi AG, et al. TRP channels in the skin. Br J Pharmacol. 2014;171:2568-2581.

38. Lumpkin EA, Caterina MJ. Mechanisms of sensory transduction in the skin. Nature. 2007;445:858-865.

39. Proksch E. Altersspezifische Prinzipien der topischen Therapie. Hautarzt. 2014;65:192-196. 